\title{
A Network Topology Control Algorithm Based on Mobile Nodes
}

\author{
https://doi.org/10.3991/ijoe.v12i10.6198 \\ Huarui $\mathrm{Wu}^{1,2,3}$, Li Zhu ${ }^{1,2,3}$ \\ ${ }^{1}$ Beijing Research Center for Information Technology in Agriculture, Beijing, China \\ ${ }^{2}$ National Engineering Research Center for Information Technology in Agriculture, Beijing, China \\ ${ }^{3}$ Key Laboratories for Agri-informatics, Ministry of Agriculture, Beijing, China
}

\begin{abstract}
Topology control is of great significance to reduce energy consumption of wireless sensor network nodes and prolong network lifetime. Different tasks taken by nodes may lead to node failures and fractures of data transmission links, hence undermining the overall network performance. In response to such problems, this paper presents a network topology control algorithm based on mobile nodes that fully considers node energy, node degree and network connectivity. Furthermore, a topology control model is established to analyze weak network topology areas and carry out local topology refactoring. Finally, a simulation experiment demonstrates that the presented algorithm is advantageous in balanced network energy consumption and network connectivity.
\end{abstract}

Index Terms-Topology control; Wireless Sensor Network; energy consumption; neighbor node set; network lifetime.

\section{INTRODUCTION}

In recent years, WSN has many important applications in such areas as national defense and military, national security, environmental monitoring, medical health and household. Network topologies, a basic part of WSN research, have laid a solid theoretical basis for research on network routing protocols, network coverage, node location, and etc. Wireless Sensor network is composed of a large amount of energy limited Sensor nodes, extend the network lifetime is the first need to consider problems. Topology control is a kind of important energy saving technology, under assuring the quality of coverage and connected, reduce the network energy consumption by reducing the redundancy link. its purpose is to maintain the topology structure, by closing some possible conflict link, or by adjusting node sends power to reduce or avoid the interference of communication nodes, and improve the network throughput.Topology control can ensure the quality of coverage and connectivity, At the same time, the topology control can reduce communication interference, improve the MAC protocol and routing efficiency, provide a topology data for data fusion; In addition, topology control can improve network reliability, scalability, and other performance. It is a problem that must be solved that is how to use effective control methods to secure reasonable topological structures which can not only prolong network lifetime under the premise of satisfying network coverage and connectivity, but give consideration to other performance such as communication jamming, network delay, load balancing, simplicity, reliability and extendibility.

As a vital research field, topology control is to provide reasonable connected paths for networks using methods like graph theory, for the purpose of save energy and adding transmission capacity. At present, research on wireless sensor network-based topology control mainly make maximizing network lifetime as a design objective, with a focus on power control and sleep scheduling. In terms of power control, some solutions have been proposed with the basic idea to prolong network lifetime by reducing transmitting power. In the CBTC algorithm [1], transmitting area of nodes is divided into non-overlapping fantype areas where a node enlarges gradually and transmits power till it finds an adjacent node or enlarges to the maximum transmitting power. The CBTC algorithm was employed to present a topology similar to Yao graph that can better decrease transmitting power of nodes [2]. Signals sent by nodes can not only be received by multiple nodes simultaneously within transmission range, but cause communication jamming $[3,4]$. Thus, each node is able to control the number of adjacent nodes and change network topologies by adjusting its own transmitting power. Kubisch et al. [5] have advanced a power control method based on node degree, in which node degree is controlled within an appropriate range via dynamic adjustment of transmitting power to reduce network energy consumption. It is, however, difficult for such algorithm to guarantee network connectivity. Wattenhofer et al. [6] have put forward the XTC algorithm that selects links by making received signal strength as a distance measure in RNG. But it is relatively simple without enough hypotheses of deployment environment or considering quality changes in communication links. Kumar et al. [7] have come up with the RIS algorithm, a simple sleep scheduling algorithm, which divides time into periods and each node independently determines whether to go into sleep at the beginning of each period. But time needs to be strictly synchronized in RIS. Heinzelman et al. [8] have proposed the LEACH algorithm that has been widely introduced. However, time needs to be strictly synchronized and uniform distribution of cluster heads fails to be ensured. Ye et al. [9] have introduced the EECS algorithm that can ensure uniform distribution of cluster heads. Like EECS and LEACH, single-hop communication of both cluster heads and sink nodes has limited network scale. Deb et al. [10] have proposed a TopDisc algorithm based on the minimum dominating set in graph theory. Ideally, clusterbased topology control is to select the least links as a backbone of network communication, while cutting costs of control and maintenance. SPN proposed by Chen et al. [11], under the premise of not undermining the previous connectivity, adaptively decides whether to serve as backbone nodes or fall asleep based on the number of remaining and adjacent nodes and node effectiveness. Sleeping 
nodes keep awake periodically to decide whether to become backbone ones, while backbone nodes periodically decide whether to withdraw. Nonetheless, with the increase of node density, SPAN exerts a worse energysaving effect. There are a variety of factors influencing network topology control which is, therefore, aimed at how to reduce network energy consumption and increase network lifetime without affecting network connectivity. In this context, this paper presents a network topology control algorithm based on mobile nodes to analyze areas with weak network links, where mobile nodes are introduced to predict potential failed links and local topology control is exercised, hence reducing global topology cost and enhancing network connectivity.

\section{TOPOLOGY CONTROL MODEL}

Supposing the wireless sensor node set $\mathrm{S}$ consisting of $\mathrm{N}$ non-overlapping nodes with a perception radius of $\mathrm{R}_{\mathrm{s}}$ is randomly deployed in a dimensional monitoring area $A$, node locations are fixed, $\mathrm{R}_{\mathrm{c}}$ is the communication radius for nodes, Rs is the maximum perception radius, and $R_{c} \geq 2 R_{s}$. A circular perception area is formed with $S_{i}\left(S_{i} \in\right.$ $\mathrm{S})$ as the center. Suppose nodes have the following properties:

(1) Sensor nodes are randomly deployed within the monitoring area with fixed locations to set up a wireless self-organizing network;

(2) With a unique ID, any two nodes within the communication radius are neighbor nodes;

(3) Communication power of nodes is adjustable, namely nodes can adjust transmitting power according to distance;

(4) Existing location algorithm can be used to collect information on network node locations;

(5) Initial energy of sensor nodes is $Q_{0}$, with a synchronous clock.

When any two nodes within the area that can communicate with each other transmit and receive data, energy consumption [12] can be expressed as:

$$
\begin{gathered}
\mathrm{Q}_{\mathrm{tx}}(\mathrm{k}, \mathrm{d})= \begin{cases}\mathrm{kQ} \mathrm{e}_{\mathrm{e}}+\mathrm{k} \varepsilon_{\mathrm{fs}} \mathrm{d}^{2}, & \mathrm{~d}<\mathrm{d}_{0} \\
\mathrm{kQ} \mathrm{e}_{\mathrm{e}}+\mathrm{k} \varepsilon_{\mathrm{mp}} \mathrm{d}^{4} & \mathrm{~d} \geq \mathrm{d}_{0}\end{cases} \\
\mathrm{Q}_{\mathrm{Rx}}(\mathrm{k}, \mathrm{d})=\mathrm{kQ} \mathrm{e}_{\mathrm{e}}
\end{gathered}
$$

where $\mathrm{Q}_{\mathrm{rx}}$ represents energy consumption for receiving data and $\mathrm{Q}_{\mathrm{tx}}$ is that for transmitting data; $\mathrm{K}$ represents the size of data package; $d$ is distance between communication nodes and $\mathrm{d}_{0}$ is reference distance (when $\mathrm{d}<\mathrm{d}_{0}$, power amplification loss employs free space propagation model, otherwise, multipath propagation model is used); and $\varepsilon_{\mathrm{fs}}$ $\varepsilon_{\mathrm{mp}}$ are parameters for power amplifiers in such two models.

Wireless signals are susceptible to external environment during transmission [13,14]. Although locations of static nodes remain unchanged, inter-node signal transmission is subject to temporal and spatial variation when network is running, making network topologies change accordingly and having some influences on network connectivity. During the running of network, differences in tasks taken by nodes give rise to failed node, thus causing link failures and increasing network energy consumption. In this context, a mobile node model is established to reasonably control the number of mobile and static nodes, realize dynamic adjustment of network topologies and strike a balance between network energy consumption and connectivity, which can effectively prolong network lifetime.

During the initialization of network, all sensor nodes, at the maximum transmitting power, identify communication relationship between nodes and create a network topology $\mathrm{G}(\mathrm{V}, \mathrm{E})$, where $\mathrm{V}$ is a collection of nodes, written as $V=\left\{v_{i} \mid v_{i} \in V\right\}$; and $\mathrm{E}$ is an inter-node frontier set, represented as $E=\left\{\left(v_{i}, v_{j}\right) \mid\left(v_{i}, v_{j}\right) \in E\right\}$. Let any intra-area node be vi, neighbor node information can be collected within the effective transmission range, with neighbor node set expressed as $\mathrm{T}\left(v_{i}\right)=\left\{v_{j} \mid\left(v_{i}, v_{j}\right) \in E, v_{j} \in V\right\}$. When the source node $\mathrm{V}_{\mathrm{i}}$ transmits data to target node $\mathrm{Vk}$, the weight for transmission path can be expressed as $W\left(v_{i}, v_{k}\right)=\sum_{j=1}^{k} W\left(v_{i}, v_{j}\right)$

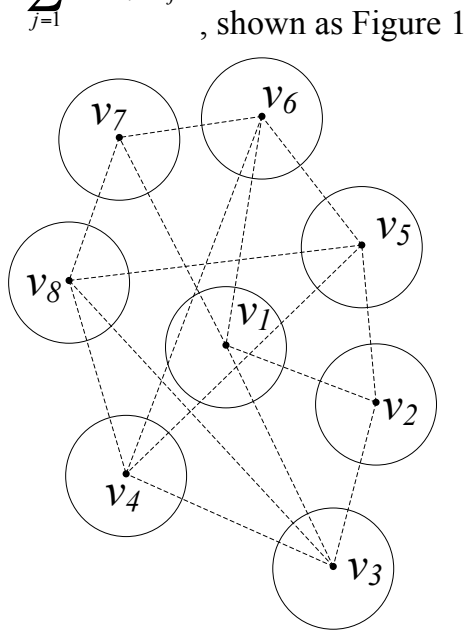

Figure 1. Initial topology

Let the number of work nodes be $\mathrm{N}(\mathrm{t})$ at time $\mathrm{t}$, path weight for vi node and any node within its neighbor set can be represented as

$$
\Gamma\left(v_{i}, v_{j}\right)=\frac{1}{k} L_{i j} \Phi\left(\alpha \frac{Q_{i}}{Q_{0 i}}, \beta \frac{Q_{j}}{Q_{0 j}}\right)
$$

where $k$ is the number of neighbor nodes of $V_{i}, Q_{j}$ is the remaining energy of $V_{i}, Q_{0 i}$ is the initial energy of $V_{i}, L_{i j}$ is the distance from $V_{i}$ to $V_{j}, \alpha$ and $\beta$ are weight factors of nodes. Network nodes take different tasks. Some nodes are confronted with bottlenecks prematurely due to heavier tasks. Node area serves as a critical topology area. The gross energy consumption of all connective areas at time $t$ can be formulated as:

$$
W(t)=\frac{L_{i j}}{\sum_{i \neq j} d_{i j}} \int_{0}^{t} \Omega(\delta, \gamma) \bullet W\left(v_{i}, v_{j}\right) d t
$$

$\mathrm{d}_{\mathrm{ij}}$ is the mean of location relationship between static nodes and mobile ones; $\mathrm{L}_{\mathrm{ij}}$ is the minimum connectivity 
path length between vi and vj; $\Omega(\delta, \gamma)$ is a distribution function for mobile and static nodes, where $\delta$ and $\gamma$ are proportionality coefficients of network sensor nodes and mobile ones respectively which can adjust the distribution balancing of network energy consumption and improve network connectivity within the range of $(0,1)$, satisfying $\delta+\gamma=1$. Considering special energy supply of mobile nodes in network topology control, we assume that there are $\mathrm{k}$ nodes in the critical topology area, then key factor $\rho_{\mathrm{i}}$ of vi can be expressed as:

$$
\rho_{i}=\frac{\eta_{i}}{\sum_{i=1}^{k} \eta_{i}} \Theta\left(W_{i}\right)
$$

where $\eta_{i}$ is node degree of vi. In light of temporal and spatial changes in network topology, such factors as total network nodes, proportional control relationship between static and mobile nodes of network sensors, location relation of mobile nodes and key node factors are transformed into linear constraints. Then, distribution relation of static nodes to mobile ones is derived by tightening constraints.

\section{TOPOLOGY CONTROL ALGORITHM}

\section{A. Related definitions}

Definition1 (Weak topology area) Let $\Lambda$ be a set of all nodes in subdomain $\mathrm{M}$, then

$$
\Lambda=\left\{v_{i} \in V: \operatorname{loc}\left(v_{i}\right) \in M\right\}
$$

There exists at least one connectivity path to $\mathrm{Vj}$ for vi in domain M, i.e., ${ }^{v_{i}->v_{j}}$; after time $\mathrm{t}, \sum_{i=1}^{n} Q_{i}-Q_{\min }<\partial$; and probability for existing intra-domain connectivity paths to face path fracture can be expressed as $P_{M \rightarrow \Lambda} \leq P_{0}$. In this case, $M$ is a weak topology area.

Definition 2 (energy topology factor) In network topology $G(V, E)$, the ratio of weight for any inter-node path $\Gamma\left(\mathrm{v}_{\mathrm{i}}, \mathrm{v}_{\mathrm{j}}\right)$ to network path weight is defined as energy topology factor for that node, which can be written as:

$$
\chi_{i j}=\frac{\Gamma\left(v_{i}, v_{j}\right)}{\sum_{\substack{i=1 \\ j=1 \\ i \neq j}}^{k} \Gamma\left(v_{i}, v_{j}\right)}
$$

Definition 3 (neighbor node set) All nodes in node set B of vi within the effective communication range are effective neighbor nodes for vi, and B is called neighbor node set.

$$
B_{i}=\left\{v_{j} \mid e\left(v_{i}, v_{j}\right) \in E, v_{i} \neq v_{j} \in V, v_{j} \in R_{i}\right\}
$$

Definition 4 (effective link set) Supposing target node vi passes information to source node $\mathrm{vj}\left(\mathrm{v}_{\mathrm{i}}->\mathrm{v}_{\mathrm{j}}\right)$, there exists at least one effective information passing path which is defined as effective link set and can be represented as:

$$
\bigcup_{\substack{v_{i} \in V \\ v_{j} \in V}} L_{v_{i}->_{v_{j}}}
$$

\section{B. Topology control algorithm based on mobile nodes}

In response to the influences of network energy balancing and network connectivity on overall network performance, this paper presents a network topology control algorithm based on mobile nodes to analyze information of adjacent nodes. Furthermore, mobile nodes are introduced to locate weak area of network links, where information on failed links are updated to reduce energy consumption and network delay resulting from unnecessary data transmission. In the process of quadratic topology building, local topology control is exercised in problem areas to reduce global topology cost and enhance network connectivity.

During the initialization of network, network nodes identify their effective neighbor nodes by transmitting information to each other at the maximum transmitting power. Let $\mathrm{M}$ be the set of information data transmitted by any sensor node vi to all network nodes, including remaining capacity of $\mathrm{Vi}$ and information on node locations. When receiving information from $\mathrm{Vi}$, vj sends to $\mathrm{Vi}$ feedback information set $\mathrm{F}$, including relevant information on vj such as power and locations. At that time, vi and vj have effective paths, weights of which are calculated. vi establishes $\mathrm{C}$, information set of its neighbor nodes, to store information related to neighbor nodes. By that analogy $\square$ each network node identifies its own neighbor node set and create the weighted undirected connected graph $\mathrm{G}^{\prime}\left(\mathrm{V}, \mathrm{E}^{\prime}\right)$ based on neighbor node information. Then, the minimum spanning tree of each node is determined according to $G^{\prime}$ edge weight. At the same time, network topology $G(V, E)$ are created under the principle of disjoint minimum path of each link., as shown in Figure 2

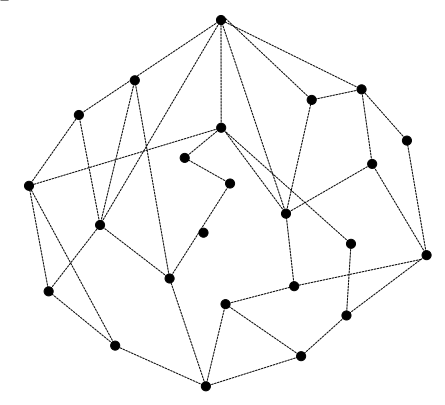

Figure 2. Topology structure

$\Gamma_{\mathrm{ij}}$ is the weight of connectivity path from vi to $\mathrm{V}_{\mathrm{j}}$ and

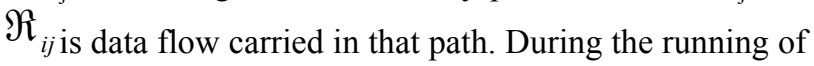
network, energy of network nodes changes with differences in tasks and transmitted data, so does weights of inter-node paths. A falling inter-node edge weight indicates decreased communication capability of the link. In case of communication between nodes that are far apart from each other, preference should be given to the path with lower link loads and higher path weights. Only in this way is it possible to take more tasks of information passing. However, fracture of adjacent links may occur to some network nodes due to excessive tasks, which undermines network connectivity and leads to unbalanced network energy distribution. To effectively avoid such cases, the topology control model is used to find $\Theta\left\{W_{k} \mid W_{k} \leq W_{0}\right\}$, a subdomain set with weak links. Supposing any subdomain is $\Theta_{i}$, key factor of $v j$ is the minimum, neighbor nodes of $\mathrm{vj}$ are centralized. If there is 
vm whose remaining capacity is less than the valve-value of node energy, then mobile node Vs is dynamically added to the joint between vj and vm; if not, mobile node vs is added at the nearest boundary point within the effective perception range. During the running of network, periodic detection is conducted on weak topology area every $\Delta t_{0}$, and recalculating edge weights within the area can total the local intra-area weight.

$$
\begin{gathered}
\Gamma\left(v_{i}, v_{j}\right)->\Gamma\left(v_{i}, v_{j}\right)+\Delta \mathrm{X} \\
\Xi\left(v_{i}\right)=\sum_{j=0}^{k} \Gamma\left(v_{i}, v_{j}\right)
\end{gathered}
$$

If $\Xi\left(v_{i}\right)$ is less than the total link weight $\Xi_{0}$, a quadratic programming is made on connectivity paths within such local area by combining current node attributes and new link paths are created to adjust intra-area topologies(seen in Figure 3). If not, there is no need for topology adjustment in the local area.

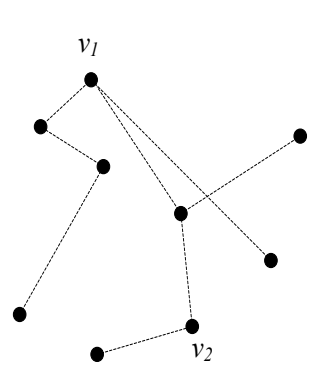

(a)

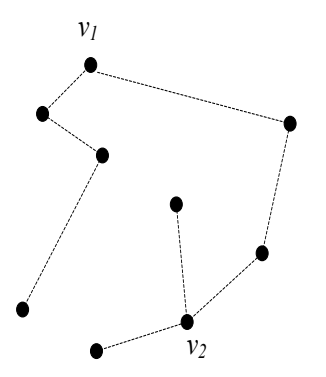

(b)
Figure 3. Local adjustments of topology

\section{SIMULATION EXPERIMENT}

In simulation experiment, the undirected graph for random node connectivity is served as the basis of wireless network topology. Connectivity between each node and its adjacent node is somewhat random. Suppose initial node energy is $100 \mathrm{~J}$ and data transmission rate is 8 packet/s, network performance parameters are tested at a periodic interval. Then, a comparison is made between the XTC algorithm and presented TCAM algorithm(seen as Figure 4).

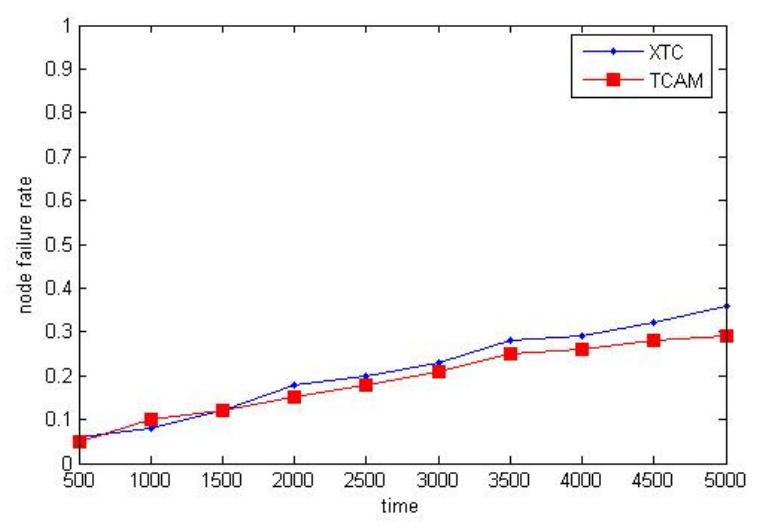

Figure 4. The network failed nodes

During the running of network, failed nodes certainly exert great impact on network performance, including connectivity, energy consumption and lifetime. For this reason, failed network nodes is evaluated based on failure rate of network nodes, as a performance index that can visually reflect network connectivity, which is the ratio of the number of failed nodes to the total nodes. As can be seen from Figure 4, there are relatively smooth changes in the number of failed nodes in the TCAM algorithm late in the operation. Besides, proportional changes in node failure are slower in comparison with XTC algorithm. A slower node failure rate or a relatively smaller number of failed nodes further demonstrates that the TCAM algorithm can reduce the number of failed nodes, cut network costs and prolong node service time.

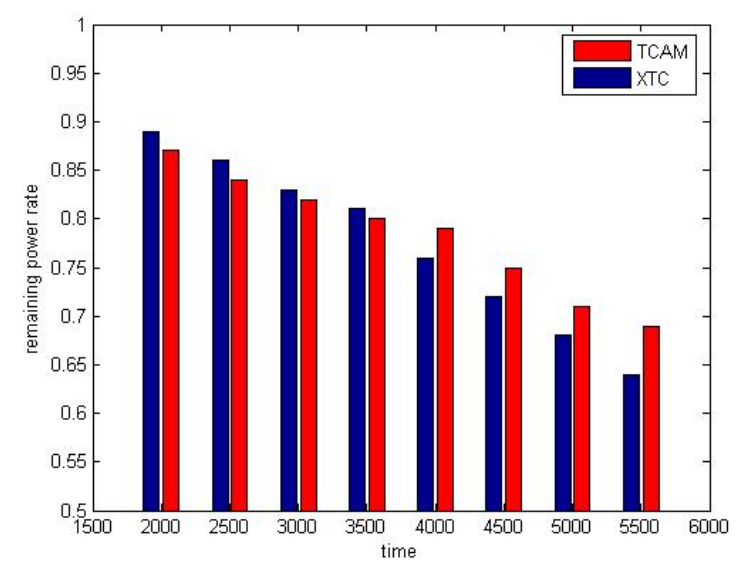

Figure 5. The network energy consumption

Energy consumption of wireless sensor network is one of the critical factor influencing the overall network performance. Network nodes work with each other. As network runs, there are some differences in remaining node energy as a result of different tasks taken by nodes. As for the TCAM algorithm, in the process of network topology, remaining energy of surrounding nodes effectively diminishes the number of failed nodes and reduces the probability of link fraction. As shown in Figure 5, energy consumption for the two algorithms is on the increase as network runs, but the TCAM algorithm has slightly lower energy consumption than the XTC algorithm.

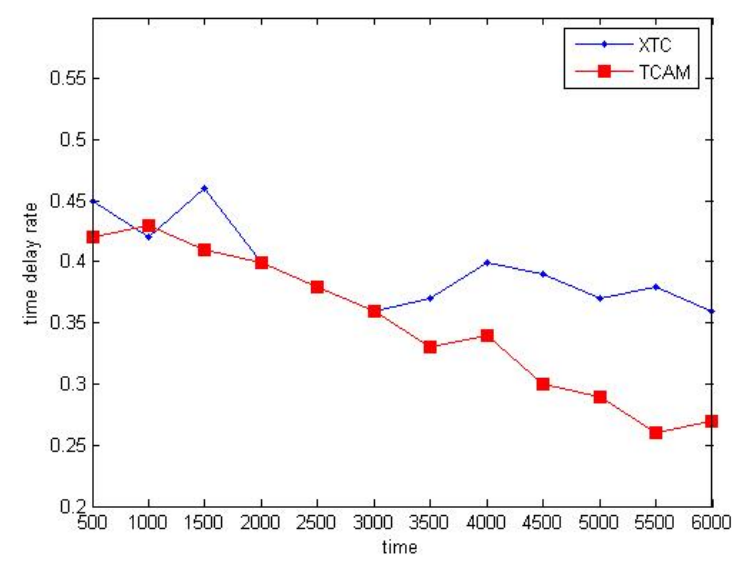

Figure 6. The network delay

As can be seen from Figure 6, the TCAM algorithm is advantageous in network delay. Energy consumption distribution of network nodes is balanced through rapid topology refactoring of local areas. In case of link fracture, rapid topology refactoring mechanism is initiated to ensure a higher success rate of network data transmission. 
As network runs, the TCAM gradually reduces network delay and later changes are relatively smooth. By contrast, the XTC algorithm has poor convergence in network delay and there are insignificant changes with the running of network.

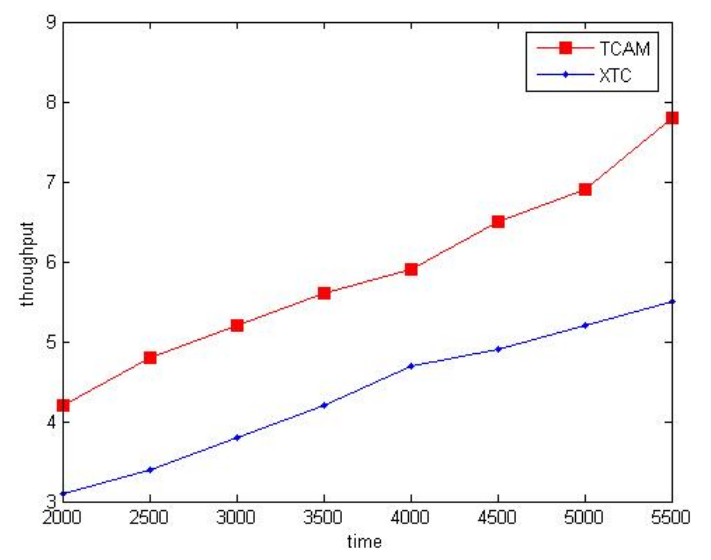

Figure 7. The network throughput

Figure 7 shows throughput changes in throughput during the running of network. As time goes on, the TCAM algorithm indicates a slightly larger increase in throughput than the XTC algorithm. Alternatively, the TCAM algorithm has a gradual rise in throughput as time goes by, which further demonstrates that the presented algorithm is not only adaptable enough to refactor topology in a short period of time, but advantageous in network data transmission with better connectivity.

\section{CONCLUSION}

With limited energy, topology control needs to optimize network connectivity, reduce node energy consumption and prolong network lifetime. This paper establishes a topology control model, and on that basis presents a network topology control algorithm based on mobile nodes to analyze network node information and identify weak topology areas with fragile links. Then, mobile nodes are introduced for local topology refactoring, which effectively improves network connectivity, reduces network energy consumption and prolongs network lifetime.

\section{REFERENCES}

[1] Li(Erran)Li,Joseph Y Halpem ,Paramvir Bahl. A conebased distributed topoplgy-control algorithm for wireless multihop networks[J].IEEE/ACM Transactions on Networking, 2005,13(1):147-159. http://dx.doi.org/10.1109/TNET.2004.84229

[2] Li L,Halpem JY,Bahl P,Wang YM,Wattenhofer R. Analysis of a cone-based distributed topology control algorithm for wireless multi-hop networks. IEEE/ACM Transactions on Networking(TON),2005,13(1):147-159

[3] Wieselthier J, Nguyen G, Ephremides A. Distributed algorithms for energy-efficient broadcasting in ad hoc networks. In: O'Berry CG, ed. Proc. of the IEEE Military Communications Conf. Piscataway: IEEE Inc., 2002. 819-824. http://dx.doi.org/10.1109/ milcom.2002.1179580

[4] Luo YH, Wang JX, Chen JE, Chen SQ. A distributed algorithm based on probability for refining energy-efficiency of multicast trees in ad hoc networks. In: Werner B, ed. Proc. of the IEEE Conf. on Local Computer Networks (LCN). New York: IEEE Inc., 2005. 482-483.
[5] Kubisch M, Karl H, Wolisz A, Zhong LC, Rabaey J. Distributed algorithms for transmission power control in wireless sensor networks. In: Yanikomeroglu H, ed. Proc. of the IEEE Wireless Communications and Networking Conf. (WCNC). New York: IEEE Press, 2003. 16-20. http://dx.doi.org/10.1109/wcnc. 2003.1200410

[6] Wattenhofer R, Zollinger A. XTC: A practical topology control algorithm for ad-hoc networks. In: Panda DK, Duato J, Stunkel C, eds. Proc. of the Int'l Parallel and Distributed Processing Symp. (IPDPS). New Mexico: IEEE Press, 2004. 216-223. http://dx.doi.org/10.1109/ipdps.2004.1303248

[7] Kumar S,Lai T H,Balogh J.On k-coverage in a mostly sleeping sensor network[A].Haas ZJ,ed.Proc. of the ACM Int'1 conf.on Mobile Computing and Networking (MobiCom)[C].New York:ACM Press,2004.144-158.

[8] Heinzelman WR, Chandrakasan AP, Balakrishnan H. EnergyEfficient communication protocol for wireless microsensor networks. In: Nunamaker J, Sprague R, eds. Proc. of the Hawaaian Int'l Conf. on System Science (HICSS). Washington: IEEE Press, 2000. 3005-3014. http://dx.doi.org/10.1109/hicss.2000.926982

[9] Ye M, Li CF, Chen GH, Wu J. EECS: An energy efficient clustering scheme in wireless sensor networks. In: Dahlberg T, Oliver R, Sen A, Xue GL, eds. Proc. of the IEEE Int'1 Workshop on Strategies for Energy Efficiency in Ad Hoc and Sensor Networks. New York: IEEE Press, 2005. 535-540.

[10] Deb B, Bhatnagar S, Nath B. A topology discovery algorithm for sensor networks with applications to network management. Technical Report, DCS-TR-441, Rutgers University, 2001.

[11] Chen B, Jamieson K, Balakrishnan H, Morris R. SPAN: An energy efficient coordination algorithm for topology maintenance in ad hoc wireless networks. ACM Wireless Networks, 2002,8(5):481-494. http://dx.doi.org/10.1023/A:1016542229220

[12] W.R. Heinzelman, A. Chandrakasan, H. Balakrishnan. Energyefficient communicationprotocol for wireless microsensor networks $[\mathrm{C}]$. Proceedings of the 33rd Annual Hawaii International Conference on System Sciences, Los Alamitos, CA, USA, 2000 http://dx.doi.org/10.1109/HICSS.2000.926982

[13] Nishiyama H, Ngo T,Ansari N, et al.On minimiaing the impact of mobility on topology control in mobile adhoc networks[J].IEEE Transactions on Wireless Communications,2012,11(3):1158-1166. http://dx.doi.org/10.1109/TWC.2012.010312.110783

[14] Chiwewe T M and Hancke G P. A distributed topology control technique for low interference and energy efficiency in wireless sensor networks[J].IEEE Transactions on Industrial Informatics,2012,8(1):11-19. http://dx.doi.org/10.1109/TII.2011.2166778

\section{AUTHORS}

Huarui Wu is a professor at National Engineering Research Center for Information Technology in Agriculture. $\mathrm{He}$ is interested in studying Artificial Intelligence. In recent years, he has participated in 18 national and provincial key scientific research projects, and published over 20 academic papers. He got the first prize of Beijing Science and Technology in 2005, and third prize of agricultural technology promotion in 2003. (wuhr@nercita.org.cn)

Li Zhu female (corresponding author), born in 1982, received her M.S. degree in Computer Applied Technology from University of Electronic Science and Technology of China (2011). Now she is reading for a doctor degree at Beijing University of Posts and Telecommunications. Her research interests include WSN and embedded system. (jolie.zhl@hotmail.com / zhul@nercita.org.cn)

The work presented in this paper was supported by the National Natural Science Foundation of China (Grants No. NSFC- 61271257), the National Natural Science Foundation of China (Grants No. NSFC- 61571051) and the National Natural Science Foundation of China (Grants No. NSFC-61471067). Submitted 03 September 2016. Published as resubmitted by the authors 05 October 2016 